\title{
DESIGN OF THE DOUBLE-LOOP NETWORKED CONTROL SYSTEM WITH DIFFERENT SAMPLING RATE BASED ON SMITH PREDICTOR
}

\author{
Hong Zhao*, Anjin Wu \\ Mechanical and control engineering college of Guilin University of Technology, Guilin, China
*Corresponding Author Email: zhaohong_glut@sina.com
}

This is an open access article distributed under the Creative Commons Attribution License, which permits unrestricted use, distribution, and reproduction in any medium, provided the original work is properly cited

\section{ARTICLE DETAILS}

\section{Article History:}

Received 02 october 2017 Accepted 06 october 2017 Available online 11 november 2017

Keywords

double closed-loop, networked control system, sampling rate, Smith predictor, network-induced delay

\section{ABSTRACT}

The double-loop networked control system with different sampling rate is investigated in this paper. In order to overcome the effect of network-induced time delay, the design methods based on conventional Smith predictor and modified Smith predictor are discussed. Simulation results based on Matlab/Simulink show that the dynamic characteristics of the system are improved due to the network-induced delay compensation, and the disturbance is restrained effectively. For the system of which the network-induced delay is random and difficult to accurately predict, the control effect of the method with modified Smith predictor is superior to the conventional Smith predictor when certain design conditions are satisfied.

\section{Introduction}

With the wide application of network control technology, more and more researchers have paid attention to study on the networked control systems (NCSs). The development of technology has been put forward higher demands for the research of rapidity, stability and accuracy of NCSs. The application of NCSs is able to enhance the flexibility of control system, reduce human resource investment, and has convenient operation and maintenance. However, the network-induced delays and packet loss in the network are inevitable because of limitation of communication protocol, bandwidth, signal conflict and so on, which greatly affect the stability and accuracy of the networked control system [1]

When sensors, controllers and actuators in two control loops are connected through the network, a double-loop NCS is formed. Double-loop NCS with different sampling rate is ubiquitous in practical industrial production sites, such as double-loop control of robot speed and position, double-loop control of servo motor's displacement and armature current, double-loop control of single-axis speed and posture of aircraft [2].

As a commonly used compensation control method in time-delay systems, Smith predictor(SP) imports an estimated compensation device into the feedback loop of a system to partially offset the network-induced delay in the feedback, so as to greatly reduce the adverse effect of the networkinduced delay on the system , which has a wide range of applications in NCS [3]. Zhang studied the NCS based on Smith predict compensation, and proposes a SP design scheme based on neuron controller [4]. Chen dealt with the long delay in the network and completed the design of SP fuzzy PI controller for the long-delay NCS [5]. Du proposed a kind of modified Smith predictor (MSP), and it is suitable for the network environment in which the network-induced delay is difficult to accurately predict [6]. Kaya proposed a design method for applying a SP to cascade control system, which improved the performance of the system but did not apply it to network control [7]. So far most of the studies on NCSs assuming all network control loops with the same sampling rate. It brings some new problems on system analysis and design while taking into account the fact that the NCSs with different sampling rate. Due to the difference of inner and outer loop's sampling rates, the fastness and stability of system are affected, so it's necessary to take optimization measures to compensate for the negative effects.

\section{DOUBLE-LOOP NCS WITH DIFFERENT SAMPLING RATE}

Figure 1 shows a common double-loop NCS structure. $R(s)$ and $C(s)$ are input and output of the system, $G_{c 1}(s)$ and $G_{c 2}(s)$ are the outer and inner loop controller respectively, $G_{p 1}(s)$ is the main controlled object, $G_{p 2}(s)$ is the deputy controlled object, $T_{1}$ is the outer loop sampling period, $T_{2}$ is the inner loop sampling period, and $F(s)$ is a disturbance signal. $e^{-\tau_{c a} s}$ is the time delay cause by network communication from controller to actuator and $e^{-\tau_{s c} s}$ is the delay from sensor to controller respectively? The pulse transfer function of the system can be written as

$$
\frac{C(z)}{R(z)}=\frac{G_{c 1}(z) G_{c 2} G_{p 2} G_{p 1}(z) z^{-\frac{\tau_{c a}}{T_{2}}}}{1+z^{-\frac{\tau_{c a}}{T_{2}}} G_{c 2} G_{p 2}(z) z^{-\frac{\tau_{s c}}{T_{2}}}+z^{-\frac{c_{c a}}{T_{1}}} G_{c 1}(z) G_{c 2} G_{p 2} G_{p 1}(z) z^{-\frac{\tau_{s c}}{T_{1}}}}
$$

Figure 1: Structure of double-loop NCS with different sampling rate Since the sampling periods of outer loop and inner loop are different and there are several delays in the denominator of Eq. (1), the system is difficult to stabilize. Therefore, the prediction model should be introduced to optimize system control.

\section{DOUBLE-LOOP NCS WITH DIFFERENT SAMPLING RATE BASED ON SP}

Now suppose the prediction model of the controlled object $G_{p 1}(s)$ and $G_{p 2}(s)$ are $G_{m 1}(s)$ and $G_{m 2}(s)$, the prediction model of network-induced delay $e^{-t_{c a} s}$ and $e^{-t_{s c} s}$ are $e^{-\tau_{c a m} s}$ and $e^{-\tau_{s c m} s}$ respectively. According to the double-loop control network structure shown in Figure 1, the SPs are added to the outer loop and the inner loop of the system respectively, as shown in Figure 2 .

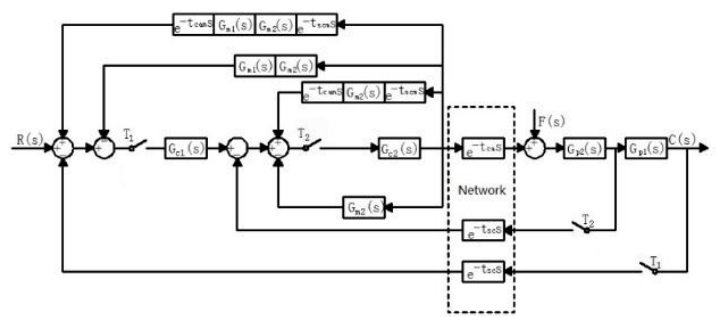

Figure 2: Structure of double-loop NCS with different sampling rate based on SP 
The pulse transfer function of the system is as follow

$$
\begin{aligned}
& \frac{C(z)}{R(z)}=\frac{G_{c 1}(z) G_{c 2} G_{p 2} G_{p 1}(z) z^{-\frac{\tau_{c o}}{T_{2}}}}{1+G_{A}+G_{B}} \\
& G_{A}=z^{-\frac{\tau_{m}}{T_{2}}} G_{c 2} G_{p 2}(z) z^{-\frac{\tau_{c m}}{T_{2}}}-z^{-\frac{\tau_{c m}}{T_{2}}} G_{c 2} G_{m 2}(z) z^{-\frac{\tau_{c m}}{T_{2}}}+G_{c 2} G_{m 2}(z) \\
& G_{B}=G_{c 1}(z)\left[z^{\frac{-\tau_{m}}{\tau_{1}}} G_{c 2} G_{p 2} G_{p 1}(z) z^{-\frac{\tau_{c k}}{T_{i}}}-z^{-\frac{\tau_{m m}}{T_{1}}} G_{c 2} G_{m 2} G_{m 1}(z) z^{\frac{\tau_{c m}}{T_{1}}}+G_{c 2} G_{m 2} G_{m 1}(z)\right]
\end{aligned}
$$

When the prediction model of the controlled object $G_{m 1}(s)$ and $G_{m 2}(s)$ and that of the inner loop delay $e^{-\tau_{\text {cam }} s}$ and $e^{-\tau_{\text {scm }} s}$ in the network achieve ideal condition: $G_{m 1}(s)=G_{p 1}(s), G_{m 2}(s)=G_{p 2}(s), \tau_{c a m}=\tau_{c a}, \tau_{s c m}=\tau_{s c}$. That is, when the prediction model and the real model are equal, the denominator with the delay link is offset, then the pulse transfer function of system can be written as

$$
\frac{C(z)}{R(z)}=\frac{G_{c 1}(z) G_{c 2} G_{p 2} G_{p 1}(z) z^{-\frac{\tau_{c a}}{T_{2}}}}{1+G_{c 2} G_{m 2}(z)+G_{c 1}(z) G_{c 2} G_{m 2} G_{m 1}(z)}
$$

Eq. (3) shows that the time delay of the closed-loop transfer function's denominator of the system is offset, and the structure of the NCS can be equivalent to

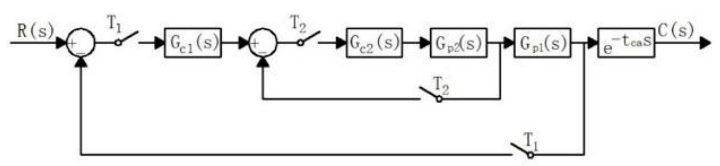

Figure 3: Structure of equivalent double-loop NCS with accurate SP model

Figure 3 demonstrate that the double-loop NCS combined with SP reduces the influence of network-induced delay on system stability. The delay in the feedback loop of the system is offset, and the network-induced delay is removed from the system transfer function's denominator and becomes a pure time-delay link. While the pure time-delay link only causes the system response delay, it does not undermine the stability of the system. However, Eq. (2) shows that such predict control requires relatively high accuracy of the predicted network-induced delay and is more suitable for the case that the network-induced delay is constant and known. While the networkinduced delay in the general NCS is usually time-varying, so it is difficult to predict the network-induced delay $e^{-\tau_{c a} s}$ and $e^{-\tau_{s c} s}$ accurately.

\section{DOUBLE-LOOP NCS WITH DIFFERENT SAMPLING RATE BASED ON MSP}

In view of the above problems, a design scheme that uses MSP [6] to compensate the double-loop NCS with different sampling rate is proposed in this paper. This compensation method does not need to predict the size of the network-induced delay, and can be applied to NCS applications of which the network-induced delay is random, time-varying, over one or even tens of sampling periods, and exists a certain amount of data packet loss. This kind of SP needs to add a prediction model of the controlled object to the controlled object port, so the modified method is suitable for situations in which the network remote environment is allowed to join the prediction model. Double-loop NCS with MSP is shown in Figure 4.

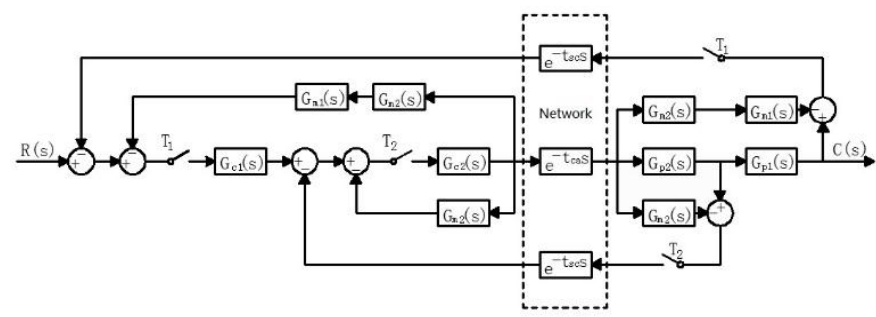

Figure 4: Structure of equivalent double-loop NCS with MSP model

The pulse transfer function of the system is as follow

$$
\begin{aligned}
& \frac{C(z)}{R(z)}=\frac{G_{c 1}(z) G_{c 2} G_{p 2} G_{p 1}(z) z^{-\frac{\tau_{a}}{T_{2}}}}{1+G_{A}+G_{B}} \\
& G_{A}=G_{c 2} G_{m 2}(z)+z^{-\frac{\tau_{m}}{T_{2}}}\left[G_{c 2} G_{p 2}(z)-G_{c 2} G_{m 2}(z)\right] z^{\frac{-\tau_{c}}{\tau_{2}}} \\
& G_{B}=G_{c 1}(z)\left[G_{c 2} G_{m 2} G_{m 1}(z)+z^{-\frac{\tau_{m s}}{T_{1}}}\left(G_{c 2} G_{p 2} G_{p 1}(z)-G_{c 2} G_{m 2} G_{m 1}(z)\right) z^{-\frac{\tau_{k}}{T_{1}}}\right]
\end{aligned}
$$

Eq. (4) shows that there is no prediction model of $e^{-\tau_{c a} s}$ and $e^{-\tau_{s c} s}$ $e^{-\tau_{\text {cam }} s}$ and $e^{-\tau_{s c m} s}$ does not appear in the denominator of transfer function, only $G_{m 1}(s)=G_{p 1}(s), G_{m 2}(s)=G_{p 2}(s)$ are needed. That is to say, Eq. (3) can be formed when the controlled object prediction model reaches the ideal condition. This means that there is no need to predict networkinduced delay after such improvement. However, such a modified method is only suitable for the case that the controlled object $G_{p 1}(s)$ and $G_{p 2}(s)$ port of the network in which the controlled object is located can be incorporated into the prediction model $G_{m 1}(s)$ and $G_{m 2}(s)$ of the controlled object.

\section{SYSTEM SIMULATION}

The software Matlab/simulink is used as simulation tool. Considering NCS shown in Figure 1, and assuming the transfer function of the controlled object $G_{p 1}(s)$ and $G_{p 2}(s)$ are $1 /(10 s+1)(s+1)^{2}$ and $1 /(30 s+1)$ respectively, the outer loop controller $G_{c 1}(s)$ adopts PI control and the proportion coefficient is $K_{\mathrm{p}}=2.681$, the integral coefficient is $T_{\mathrm{i}}=0.045$, the inner loop controller $G_{c 2}(s)$ adopts P control, and proportion coefficient is $K_{\mathrm{p}}=0.05$, the networkinduced delays $\tau_{c a}$ and $\tau_{s c \text { I }}$ are $3 \mathrm{~s} . T_{1}$ is the sampling period of the outer loop and $T_{2}$ is the sampling period of the inner loop.

Compare the double-loop NCS with SP shown in Figure 2 and that without SP shown in Figure 1. Assuming the network-induced delays are known, and $\tau_{c a \mathrm{~m}}=\tau_{c a} \tau_{s c \mathrm{~m}}=\tau_{s c}$. Let $T_{1}=15 \mathrm{~s}$ and $T_{2}=1 \mathrm{~s}$, the response curves can be obtained as shown in Figure 5. Y1 is the unit step response of the doubleloop NCS with different sampling rate based on SP, and Y2 is the unit step response of the normal double-loop NCS with different sampling rate. As shown from the Figure 5, the dynamic control performance of unit step response curve $\mathrm{Y} 1$ is better than that of $\mathrm{Y} 2$

Two unit step disturbances are respectively added to the two systems at $t$ $=350 \mathrm{~s}$, the response curves as shown in Figure 6, which indicates that the NCS with SP has better fastness, stability and anti-interference ability.

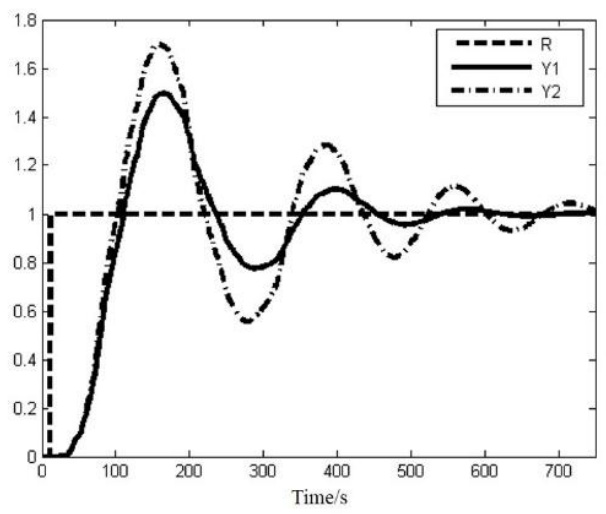

Figure 5: Comparison of the NCS with and without SP $\left(T_{1}=15 \mathrm{~s}, T_{2}=1 \mathrm{~s}\right)$

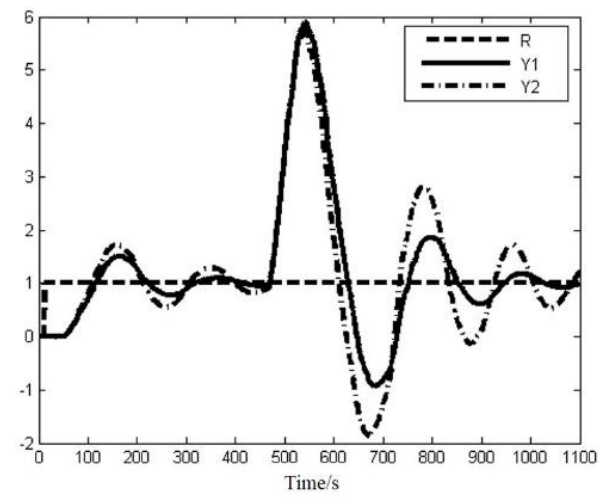

Figure 6: Comparison of the NCS with and without SP $\left(T_{1}=15 \mathrm{~s}, T_{2}=1 \mathrm{~s}\right)$

For comparing the effect of conventional SP and MSP for double-loop NCS with different sampling rate, keep $T_{2}=1 \mathrm{~s}$, and change $T_{1}$ as $10 \mathrm{~s}, 15 \mathrm{~s}$ and $25 \mathrm{~s}$ respectively. The unit step response of two systems is shown in Figure 7, 8, and 9. It is shown that as the ratio of the inner and outer ring sampling rates increases , the rise time and adjustment time of the curve $\mathrm{Y} 1$ are shorter 
and the overshoot is smaller, which shows that dynamic characteristics of the NCS with MSP has a better improvement.

When the network-induced delays fluctuate randomly in a large range and difficult to accurately predict, the compensation effect of SP shown in Figure 2 will be weakened, and the system with MSP shown in Figure 3 will not be affected. Change the fluctuation range of $\tau_{c a}$ and $\tau_{s c}$ from $1 \mathrm{~s}$ to $9 \mathrm{~s}$ randomly, while the SP in Figure 2 takes an average delay $\tau_{\text {cam }}=\tau_{\text {scm }}=5$. Setting $T_{1}=2 \mathrm{~s}$ and $T_{2}=1 \mathrm{~s}$ and the response curves are shown in Figure 10. Compared with Figure 7, it can be seen that the double-loop NCS with MSP is not affected by the increase of variation range of the delay, while the dynamic performance of the double-loop NCS with the conventional SP is significantly deteriorated.

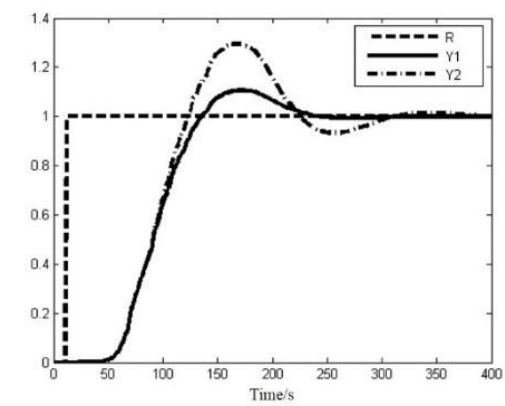

Figure 7: Comparison of MSP with SP $\left(T_{1}=10 \mathrm{~s}, T_{2}=1 \mathrm{~s}\right)$

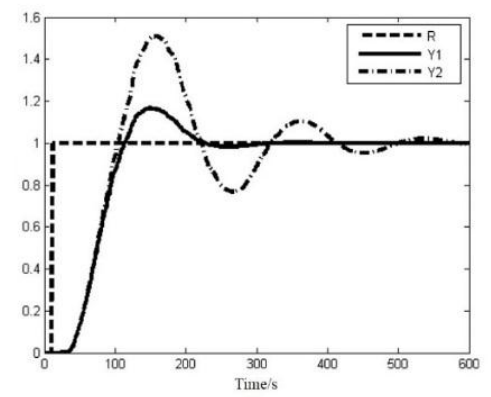

Figure 8: Comparison of MSP with SP $\left(T_{1}=15 \mathrm{~s}, T_{2}=1 \mathrm{~s}\right)$

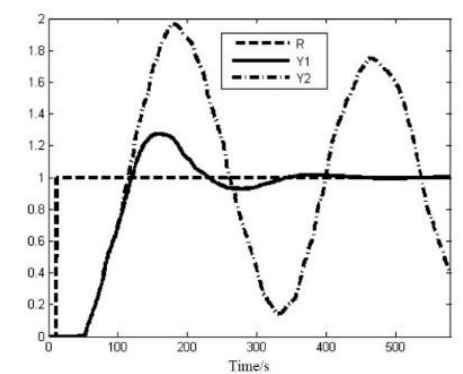

Figure 9: Comparison of MSP with SP $\left(T_{1}=25 \mathrm{~s}, T_{2}=1 \mathrm{~s}\right)$

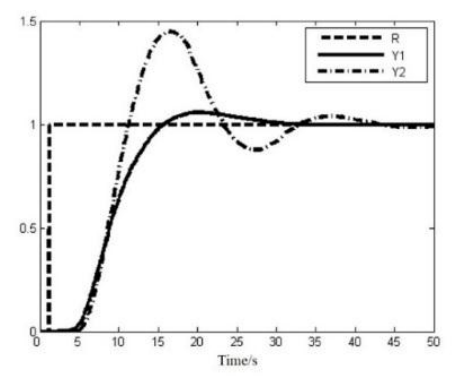

Figure 10: Comparison of MSP with SP when the network-induced delay randomly fluctuates from $1 \mathrm{~s}$ to $9 \mathrm{~s}\left(T_{1}=2 \mathrm{~s}, T_{2}=1 \mathrm{~s}\right)$

\section{CONCLUSION}

In summary, when applied to double-loop NCS with different sampling rate, SP can be used to overcome the impact of network-induced delay on the system. The simulation results show that when the prediction model can be added into the part of controlled object, for those NCSs whose network-induced delay is random, time-varying and difficult to accurately predict, the control method with MSP is more effective than the conventional SP method since it is not necessary to predict the size of the network-induced delay.

\section{ACKNOWLEDGMENTS}

This work was financially supported by the Scientific Research Project of Guangxi Colleges and Universities (No. YB2014145).

\section{REFERENCES}

[1] Zhang, W., Branieky, M.S., Phillips, S.M. 2016. Stability of Networked Control System. IEEE Control System Magazine, 21 (l), 84-99.

[2] Gu, C., Liu, D., Zhao, H., Lian, Y. 2013. Research on Modeling and Stability of a Class of Different Sampling Rates of SISO Network Control System. Manufacturing Automation, 35 (6), 36-40.

[3] Smith, 0.J.M. 1957. Close Controller of Loops with Dead Time. Chemical Engineering Progress, 53 (5), 217-219.

[4] Zhang, H., Li, Z. 2012. Simulation of Networked Control System Based on Smith Prediction Compensation. Computer Engineering and Application, 48 (8), 243-245.

[5] Chen, H., Guan, Q., Wang, W. 2005. Design of a Fuzzy PI Controller with Smith Predictor for Networked Control Systems with Long Time Delay. Journal of Zhejiang University of Technology, 33 (4), 418-420+479.

[6] Du, F., Qian Q., Du, W. 2000. Networked Control Systems Based on New Smith Predictor. Journal of Southwest Jiaotong University, 45 (1), $65-$ $69+81$.

[7] Kaya, I. 2001. Improving Performance Using Cascade Control and a Smith Predictor. ISA Transactions, 40 (3), 223-234 